\title{
Hybrid endoscopic mucosal resection and full-thickness resection for large colonic polyps harboring a small focus of invasive cancer: a case series
}

\section{두)(요우}

Authors

Jamie S. Chua*, 1, Hao Dang*, 1, Liselotte W. Zwager², Nik Dekkers' ${ }^{1}$, James C. H. Hardwick ${ }^{1}$, Alexandra M. J. Langers' Jolein van der Kraan' ${ }^{1}$, Lars E. Perk ${ }^{3}$, Barbara A. J. Bastiaansen², Jurjen J. Boonstra ${ }^{1}$, on behalf of the Dutch eFTR Working Group

Institutions

1 Department of Gastroenterology and Hepatology, Leiden University Medical Center, Leiden, the Netherlands

2 Department of Gastroenterology and Hepatology, Amsterdam University Medical Center, Amsterdam, the Netherlands

3 Department of Gastroenterology and Hepatology, Haaglanden Medical Center, the Hague, the Netherlands

submitted 13.3.2021

accepted after revision 8.6 .2021

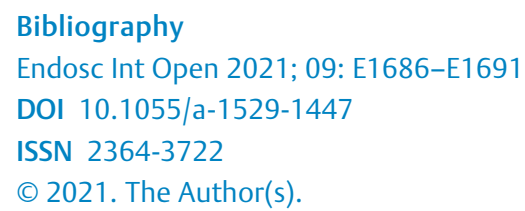

P.O. Box 9600, 2300 RC Leiden, The Netherlands

Fax: +31-71-5268115

h.dang@lumc.nl

\section{ABSTRACT}

Endoscopic treatment of large laterally spreading tumors (LSTs) with a focus of submucosally invasive colorectal cancer (T1 CRC) can be challenging. We evaluated outcomes of a hybrid resection technique using piecemeal endoscopic mucosal resection (pEMR) and endoscopic full-thickness resection (eFTR) in patients with large colonic LSTs containing suspected T1 CRC. Six hybrid pEMR-eFTR procedures for T1 CRCs were registered in a nationwide eFTR registry between July 2015 and December 2019. In all cases, the invasive part of the lesion was successfully isolated with eFTR; with eFTR, histologically complete resection of the invasive part was achieved in $5 / 6$ patients $(83.3 \%)$. No adverse events occurred during or after the procedure. The median follow-up time was 10 months (range 6-27), with all patients having undergone $\geq 1$ surveillance colonoscopy. One patient had a small adenomatous recurrence, which was removed endoscopically. In conclusion, hybrid pEMR-eFTR is a promising noninvasive treatment modality that seems feasible for a selected group of patients with large LSTs containing a small focus of T1 CRC.

\section{Introduction}

Although endoscopic full-thickness resection (eFTR) has been shown to be effective in resecting colorectal tumors [1], its applicability remains limited due to the size restrictions of the eFTR devices. To overcome this issue, several Western endoscopists have recently proposed combining eFTR with other treatment modalities (e.g. snare polypectomy [2] or piecemeal endoscopic mucosal resection [pEMR] [3-5]). These hybrid techniques may be useful when resecting large and complex colorectal tumors such as nonlifting adenomas with a diameter of $>20 \mathrm{~mm}[3,5]$. Here, we describe a series of six patients with colonic laterally spreading tumors (LSTs) containing a small $(<20 \mathrm{~mm})$ focus of submucosally invasive colorectal cancer (T1 (RC), treated in a single session using hybrid pEMR-eFTR.

\footnotetext{
* These authors contributed equally to this work.
} 


\section{Case series}

Patients with a histological diagnosis of T1 CRC who underwent hybrid pEMR-eFTR were selected from a nationwide multicenter eFTR registry (NTR6292, http://www.trialregister.nl/). Six patients with a large LST containing suspected T1 CRC were treated with hybrid pEMR-eFTR ( judged as having one well-demarcated focus of T1 CRC based on a Kudo Vi pit pattern, with a median size of $12.5 \mathrm{~mm}$ (range 5-20). Indications for hybrid pEMR-eFTR were as follows: two patients were considered inoperable due to comorbidity and/ or frailty; two patients refused surgery, both had undergone oncological surgery (sigmoid resection, low anterior resection) for CRC in the past; and two patients strongly preferred endoscopic treatment above surgical resection after extensive discussion with their physician. For all patients, endoscopic submucosal dissection (ESD) was not considered feasible by the treating endoscopist, mainly because of the relatively high risk of perforation [6, 7].

Target lesions were identified and carefully inspected using high definition, white light endoscopy (Olympus HQ190, Tokyo, Japan) and magnifying virtual chromoendoscopy in order to distinguish polyp parts with benign features (Kudo IIIS/L and IV; Sano II) from areas with invasive features (Kudo Vi; Sano IIla). A straight transparent cap (Olympus) was used for inspection and to measure the size of the invasive part of the polyp. Hybrid pEMR-eFTR was started with submucosal injection and snare polypectomy of the benign parts of the polyp in order to carefully isolate the invasive part of the polyp ( $\triangleright$ Fig.1). The pEMR-resected polyp tissue parts were collected and sent for histopathologic analysis. Next, the colonoscope was withdrawn, the eFTR device (Ovesco Endoscopy AG, Tübingen, Germany) was mounted onto the endoscope, and eFTR of the suspected invasive part was performed according to standard procedures [1]. The resected eFTR specimen was retrieved, stretched out, and pinned down on cork before immersion into formalin. Finally, the scope was reintroduced to examine clip positioning. Procedure time was defined as the time between first introduction and last withdrawal of the endoscope (including the pEMR part, withdrawal for mounting the eFTR device, reintroduction and subsequent eFTR, withdrawal when retrieving the eFTR specimen, and reintroduction to examine clip positioning). Endoscopically resected specimens were sectioned serially at 2-mm intervals and embedded for histologic examination. The procedure was deemed successful when there was no histologic evidence of invasive growth among the pEMR-resected tissue parts and when evaluation of the eFTR resection specimen confirmed that the vertical and lateral resection margins of the invasive part were tumor free.

The median procedure time was 84.5 minutes (range 58140). In all procedures, the endoscopist managed to isolate the invasive part by $\mathrm{pEMR}$ and perform a technically successful eFTR ( Fig.2). There were no immediate or delayed adverse events (e.g. bleeding, perforation). All patients stayed one night in the hospital for observation and were discharged the next day. All eFTR specimens contained a focus of histolo-

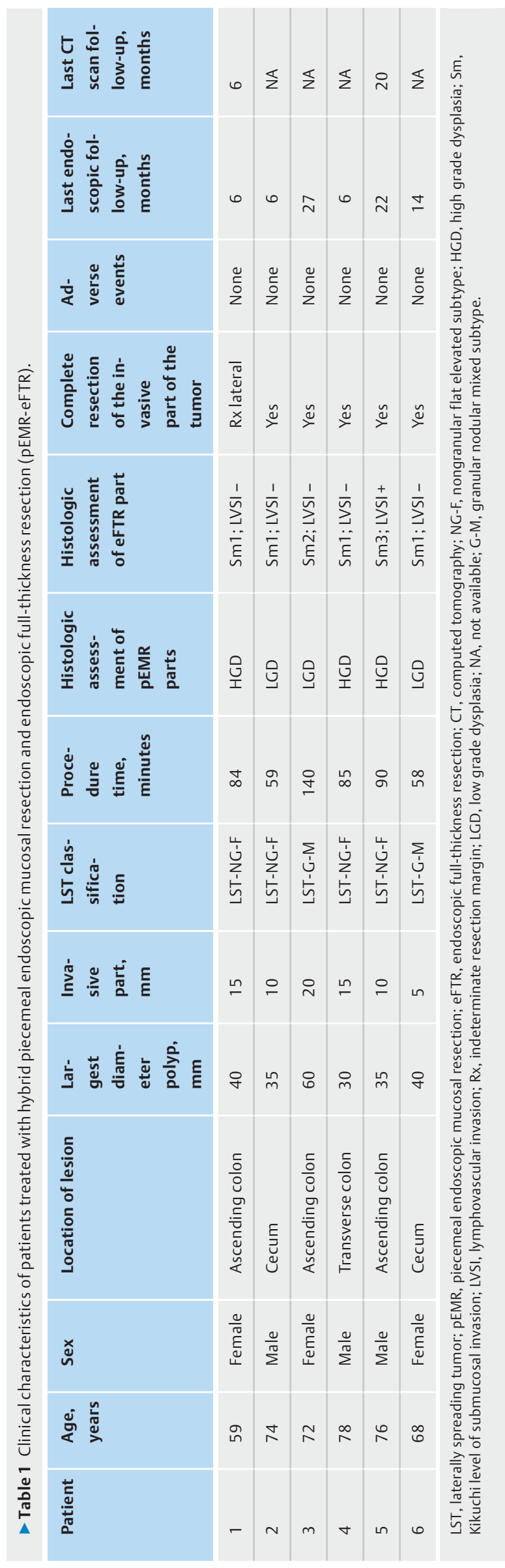




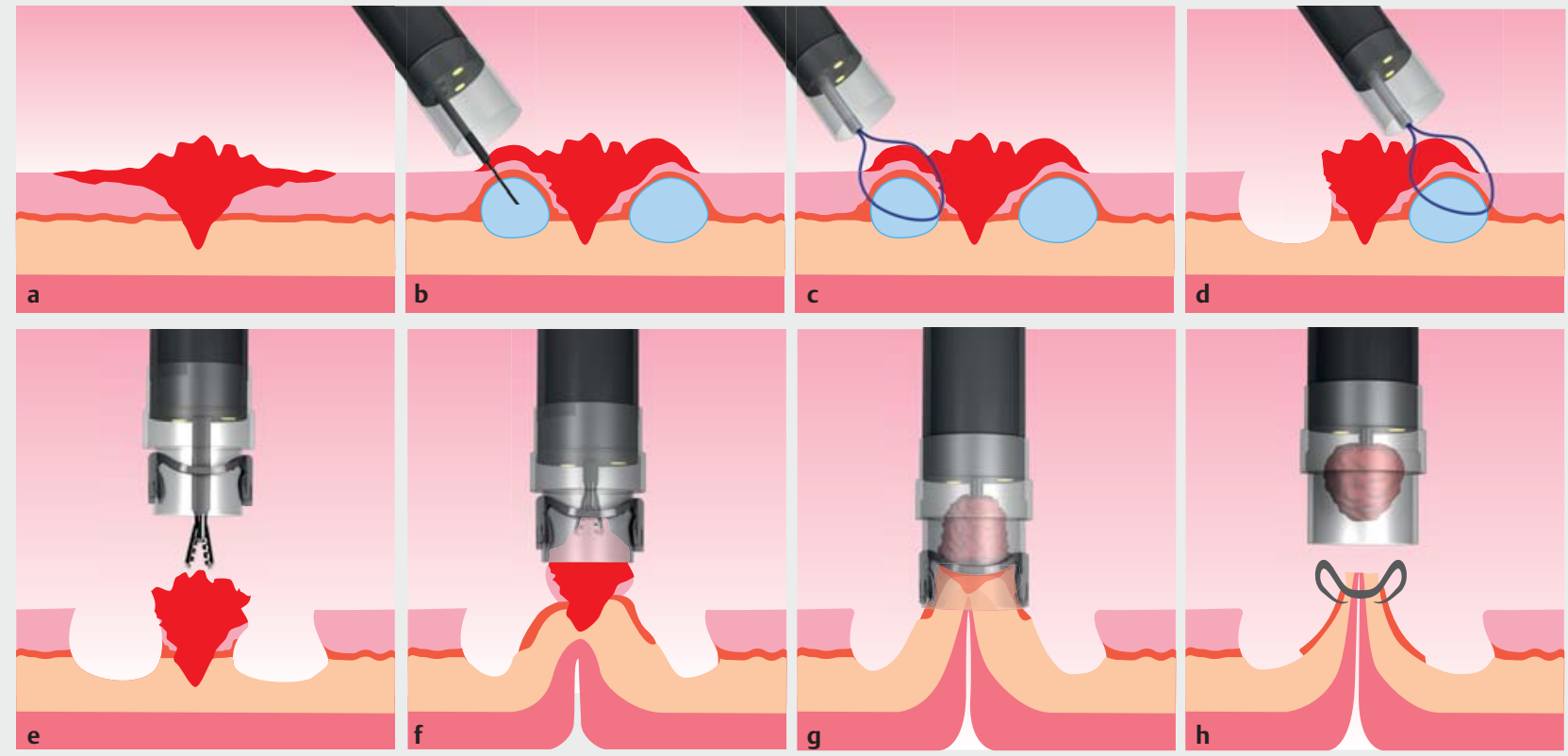

- Fig. 1 Schematic illustration of the hybrid piecemeal endoscopic mucosal resection and endoscopic full-thickness resection (pEMR-eFTR) technique for a large colonic laterally spreading tumor (LST) with a small focus of T1 colorectal cancer. a A large (>20 mm) LST was observed in the colon with a small region $(<20 \mathrm{~mm})$ containing malignant features. b A mixture of saline, epinephrine, and methylene blue was injected into the submucosa. $\mathbf{c}$ pEMR of the benign part was performed according to standard procedures and repeated until the invasive part was isolated (d). e Next, the full-thickness resection device was mounted on top of the endoscope. $\mathbf{f}$ The lesion was grasped with forceps and pulled into the cap entirely $(\mathbf{g})$. $\mathbf{h}$ Finally, in a single step, an over-the-scope clip was deployed and a pre-loaded snare resected the tissue above the clip, leaving an automatically sealed wall defect.
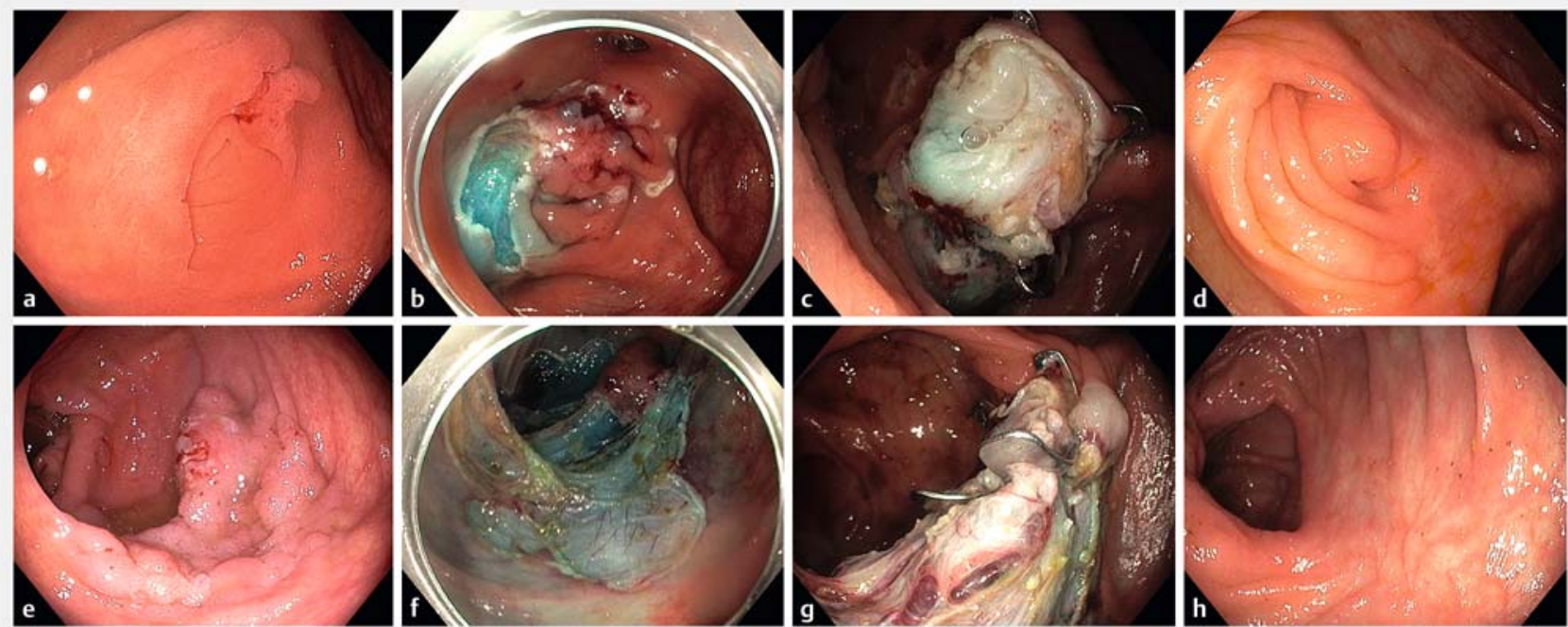

- Fig. 2 Endoscopic images of hybrid piecemeal endoscopic mucosal resection and endoscopic full-thickness resection (pEMR-eFTR). Endoscopic images of patients 2 (a-d) and 3 (e-h). a, e Endoscopic image of the lesion. b, f Isolation of the invasive part after piecemeal endoscopic resection of the noninvasive parts. $\mathbf{c , g}$ After eFTR of the invasive part. $\mathbf{d}, \mathbf{h}$ The scar at the last follow-up colonoscopy.

gically confirmed T1 CRC, and pathological assessment showed complete resection of the invasive part in $5 / 6$ cases (83.3\%; Fig. 3 ). In the remaining case, one of the lateral margins of the invasive part could not reliably be assessed owing to cauterization artefacts; the basal resection margin was tu- mor free. The adjacent pEMR-resected tissue parts showed low grade dysplasia in three cases and high grade dysplasia in the remaining three; none of the pEMR-resected tissue parts contained invasive cancer. 

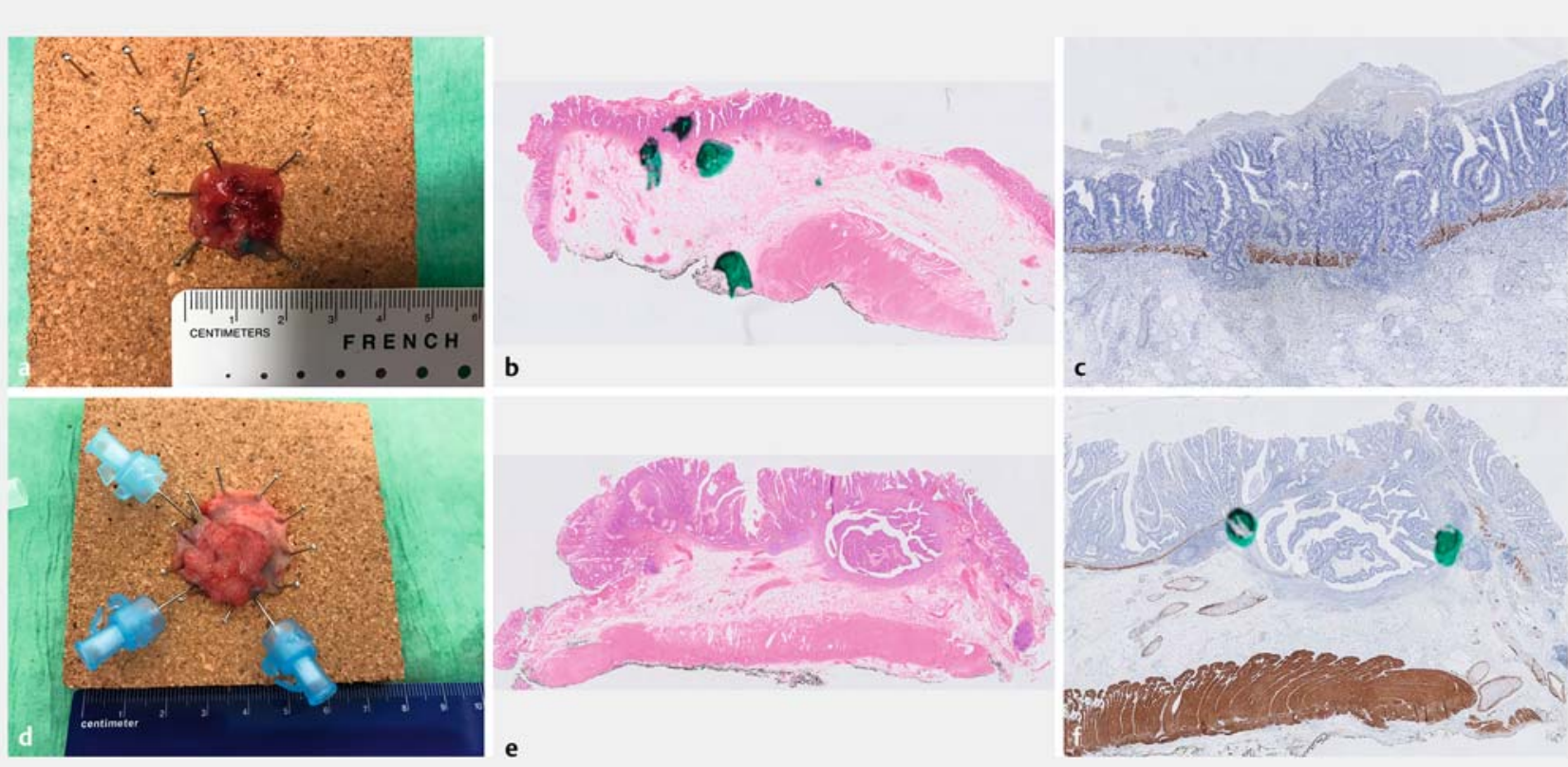

Fig. 3 Pathological images of hybrid piecemeal endoscopic mucosal resection and endoscopic full-thickness resection (pEMR-eFTR). Pathological images of patients $2(\mathbf{a}-\mathbf{c})$ and $\mathbf{3}(\mathbf{d}-\mathbf{f})$. a, d The eFTR specimen pinned onto cork. $\mathbf{b}, \mathbf{e}$ Hematoxylin and eosin staining of the eFTR specimen. $\mathbf{c}, \mathbf{f}$ Desmin staining of the eFTR specimen, with interruption of the muscularis mucosae.

After pEMR-eFTR, all patients were discussed in a multidisciplinary meeting. The patient with an Rx resection of the invasive part opted for endoscopic surveillance instead of a right-sided colectomy. The patient with deep submucosal invasion (Sm3) and lymphovascular invasion was already deemed inoperable beforehand and thus underwent endoscopic surveillance. The median follow-up duration was 10 months (range $6-27)$. All patients underwent a surveillance colonoscopy after 6 months. One patient had local recurrence of low grade adenomatous tissue at the 6-month follow-up, which was successfully treated with endoscopic resection; a surveillance colonoscopy 8 months later showed a clear polypectomy scar without signs of recurrence. Two patients underwent an additional surveillance colonoscopy after 22 and 27 months, respectively; none of them had signs of local recurrence. Additional imaging with abdominothoracic computed tomography was performed in two patients at 6 and 20 months, respectively, and neither showed signs of local recurrence or distant metastases.

\section{Discussion}

This case series demonstrates that hybrid pEMR-eFTR seems to be feasible for large colonic LSTs with a small focus of T1 CRC, with a success rate of $83 \%$ and satisfactory short-term oncological outcomes in our experience. To our knowledge, this is the first prospective series of patients with invasive tumors to be treated with hybrid pEMR-eFTR.

Currently, ESD is the only other resection technique that is suitable for treating large colonic tumors containing T1 CRC in a noninvasive manner, but its use is not yet widespread (i.e. outside Asian countries). Owing to a lack of opportunities to ac- quire sufficient skill (e.g. structured training programs, suitable starting cases), only a few Western endoscopists are able to perform colonic ESD [8], and their performance considerably lags behind that of Asian colleagues [9]. Data from several Western endoscopy centers suggest that right-sided ESD in particular can be quite hazardous, with adverse events occurring in $>20 \%$ of patients $[9,10]$ compared with $<5 \%$ at Asian centers $[6,9,11]$. In contrast, the use of eFTR is much more common in the West, and adverse event rates seem to be considerably lower when compared with right-sided ESD at Western centers (10\% vs. > $20 \%$ ) [1, 12-15]. In our series, hybrid pEMR-eFTR was therefore primarily used for tumors located in the right hemicolon. Although our sample size was not large enough to demonstrate complete safety of this technique, our results suggest that hybrid pEMR-eFTR for invasive colon tumors seems to be safe, as no adverse events occurred during or after the procedure. In line with our data, a recent study on hybrid pEMR-eFTR for noninvasive colorectal polyps also reported an acceptable overall adverse event rate of $3.2 \%$ (1/31) [5]. Another advantage of the hybrid technique is that tumors with deep submucosal invasion (i.e.>Sm1) can be resected with adequate vertical resection margins. This can be more difficult to achieve with ESD because this method requires dissection in the submucosal plane. As several studies show that deep submucosal invasion appears not to be an independent risk factor for distant disease $[16,17]$, maximizing the chance of achieving complete resection of deeply invasive T1 CRCs becomes especially important, as this may be considered curative in some cases.

A major concern of hybrid pEMR-eFTR is that the invasive part of the tumor may not be completely resected by eFTR but 
may be removed in a piecemeal fashion. This could be problematic because piecemeal resection of T1 CRCs often require additional surgical treatment owing to suboptimal assessment of histological high risk features [16]. As hybrid pEMR-eFTR relies heavily on accurate identification of noninvasive and (suspected) invasive areas, an important prerequisite for performing this technique is careful optical assessment of the lesion. In our series, we were able to successfully distinguish noninvasive and invasive tumor parts in all six patients using virtual chromoendoscopy. Although advanced imaging modalities have been shown to provide adequate diagnostic accuracy [16], it is important to note that even in expert hands, around $10 \%$ of invasive cancers may still be missed [18]. However, especially for patients with right-sided tumors, we consider this risk to be acceptable as the only feasible alternative at most Western centers would often be invasive surgery. Next, one should be aware that some T1 CRCs may show multifocal invasion [19]. If the eFTR-resected tissue part does not contain all the invasion sites, the situation would be the same as if the tumor were resected piecemeal. Therefore, we believe that hybrid pEMR-eFTR should be mainly used for large LSTs (i.e. $>20 \mathrm{~mm}$ ) containing only one small (i. e. $<20 \mathrm{~mm}$ ) and well-demarcated area of suspected T1 CRC that could fit into the eFTR cap with a broad margin of optically benign adenomatous tissue. In particular, hybrid pEMR-eFTR should be avoided for pseudodepressed nongranular LSTs, as these tumors have a substantially higher risk of multifocal invasion (46.9\%) compared with other subtypes such as flat-elevated, nongranular LSTs (11.8\%) and granular nodular mixed LSTs (7.9\%) [19]. Finally, it is important to keep in mind that short-term local recurrence occurs in a substantial proportion of patients $(\sim 20 \%)$ treated with piecemeal resection techniques [20]. Our data suggest that the same applies to patients treated with hybrid pEMReFTR, with $1 / 6$ patients (16.7\%) experiencing local adenomatous recurrence after 6 months. This warrants timely endoscopic follow-up after hybrid pEMR-eFTR. Unfortunately, the long-term oncological outcomes of hybrid pEMR-eFTR remain largely unclear, as only two patients from our series had more than 2 years of follow-up.

In conclusion, our case series suggests that hybrid pEMReFTR is a feasible treatment modality for large colonic LSTs with a small focus of suspected T1 CRC. Hybrid pEMR-eFTR may be particularly promising for patients with tumors located in the right hemicolon, who are at high risk of adverse events from surgery (owing to comorbidity or frailty) as well as from ESD (owing to a lack of sufficient expertise, which is often the case at non-Asian endoscopy centers). Larger sample sizes and long-term follow-up data are needed to determine the efficacy and safety of this resection technique.

\section{Acknowledgment}

The Dutch eFTR Working Group: Prof. dr. P. Fockens; Prof. dr. E. Dekker; Drs. B.A.J. Bastiaansen; Drs. B.W. van der Spek; Dr. R.M. Schreuder; Dr. L.E. Perk; Dr. J.J. Boonstra; Prof. dr. B.L.A.M. Weusten; Dr. H. van der Sluis; Dr. H.J. Wolters; Dr. F.C. Bekkering; Dr. S.T. Rietdijk; Dr. M.P. Schwartz; Prof. dr. W.B. Nagen- gast; Dr. W.R. ten Hove; Dr. J.S. Terhaar sive Droste; Dr. F.J. Rando Munoz; Drs. M.S. Vlug; Dr. H. Beaumont; Dr. M.H.M.G. Houben; Dr. T.C.J. Seerden; Dr. T.R. de Wijkerslooth; Dr. E.A.R. Gielisse; Dr. Y. Hazewinkel; Dr. R.J.J. de Ridder; Dr. J.W.A. Straathof. The Dutch eFTR Registry received an unrestricted grant from Ovesco Endoscopy AG for database costs. Ovesco Endoscopy AG was not involved in the design, collection, analysis, or interpretation of data from the Dutch eFTR registry. No other funding was received for the Dutch eFTR registry, and no endoscopic equipment was donated by the manufacturer.

\section{Competing interests}

Jurjen J. Boonstra is a consultant at Boston Scientific. Barbara A. J. Bastiaansen has received speaker fees from Olympus, Tillotts Pharma AG, and Ovesco Endoscopy AG. All other authors declare that they have no conflict of interest.

\section{References}

[1] Zwager LW, Bastiaansen BA], Bronzwaer MES et al. Endoscopic fullthickness resection (eFTR) of colorectal lesions: results from the Dutch colorectal eFTR registry. Endoscopy 2020; 52: 1014-1023

[2] Fischer A, Knoop RF, Walker C et al. Resection of a large rectal polyp with the simultaneous combination of snare polypectomy and fullthickness resection device resection. Endoscopy 2015; 47: E607-608

[3] Meier B, Caca K, Schmidt A. Hybrid endoscopic mucosal resection and full-thickness resection: a new approach for resection of large nonlifting colorectal adenomas (with video). Surg Endosc 2017; 31: 4268-4274

[4] Zimmer V. Hybrid precutting EMR-EFTR resection for a tricky rectal adenoma recurrence with high-grade fibrosis. Dig Liver Dis 2020; 52 : 681-682

[5] Mahadev S, Vareedayah AA, Yuen S et al. Outcomes of a hybrid technique using EMR and endoscopic full-thickness resection for polyps not amenable to standard techniques (with video). Gastrointest Endosc 2021: doi:10.1016/j.gie.2021.02.009

[6] Imai K, Hotta K, Yamaguchi Y et al. Preoperative indicators of failure of en bloc resection or perforation in colorectal endoscopic submucosal dissection: implications for lesion stratification by technical difficulties during stepwise training. Gastrointest Endosc 2016; 83: 954962

[7] Saunders BP, Tsiamoulos ZP. Endoscopic mucosal resection and endoscopic submucosal dissection of large colonic polyps. Nat Rev Gastroenterol Hepatol 2016; 13: 486-496

[8] Wang AY, Draganov PV. Training in endoscopic submucosal dissection from a Western perspective. Tech Gastrointest Endosc 2017; 19: 159-169

[9] Fuccio L, Hassan C, Ponchon T et al. Clinical outcomes after endoscopic submucosal dissection for colorectal neoplasia: a systematic review and meta-analysis. Gastrointest Endosc 2017; 86: 74-86

[10] Ronnow CF, Uedo N, Toth E et al. Endoscopic submucosal dissection of 301 large colorectal neoplasias: outcome and learning curve from a specialized center in Europe. Endosc Int Open 2018; 6: E1340-E1348

[11] Takeuchi Y, lishi H, Tanaka S et al. Factors associated with technical difficulties and adverse events of colorectal endoscopic submucosal dissection: retrospective exploratory factor analysis of a multicenter prospective cohort. Int J Colorectal Dis 2014; 29: 1275-1284 
[12] Li P, Ma B, Gong S et al. Efficacy and safety of endoscopic full-thickness resection in the colon and rectum using an over-the-scope device: a meta-analysis. Surg Endosc 2021; 35: 249-259

[13] Meier B, Stritzke B, Kuellmer A et al. Efficacy and safety of endoscopic full-thickness resection in the colorectum: results from the German Colonic FTRD Registry. Am J Gastroenterol 2020; 115: 1998-2006

[14] Kuellmer A, Mueller ], Caca K et al. Endoscopic full-thickness resection for early colorectal cancer. Gastrointest Endosc 2019; 89: 1180-1189

[15] Schmidt A, Beyna T, Schumacher B et al. Colonoscopic full-thickness resection using an over-the-scope device: a prospective multicentre study in various indications. Gut 2018; 67: 1280-1289

[16] Rex DK, Shaukat A, Wallace MB. Optimal management of malignant polyps, from endoscopic assessment and resection to decisions about surgery. Clin Gastroenterol Hepatol 2019; 17: 1428-1437
[17] Kouyama Y, Kudo SE, Miyachi $\mathrm{H}$ et al. Practical problems of measuring depth of submucosal invasion in T1 colorectal carcinomas. Int J Colorectal Dis 2016; 31: 137-146

[18] Barendse RM, Musters GD, de Graaf EJR et al. Randomised controlled trial of transanal endoscopic microsurgery versus endoscopic mucosal resection for large rectal adenomas (TREND Study). Gut 2018; 67: 837-846

[19] Ishigaki T, Kudo SE, Miyachi H et al. Treatment policy for colonic laterally spreading tumors based on each clinicopathologic feature of 4 subtypes: actual status of pseudo-depressed type. Gastrointest Endosc 2020; 92: 1083-1094

[20] Belderbos TD, Leenders M, Moons LM et al. Local recurrence after endoscopic mucosal resection of nonpedunculated colorectal lesions: systematic review and meta-analysis. Endoscopy 2014; 46: 388-402 